\title{
Frequência, Duração, Abrangência Espacial e Intensidade das Ondas de Calor no Brasil
}

\author{
Daniel Pires Bitencourt ${ }^{1}$, Márcia Vetromilla Fuentes ${ }^{2}$, Paulo Alves Maia ${ }^{3}$, \\ Fabiano Tigueiro Amorim ${ }^{4}$ \\ ${ }^{1}$ Centro Estadual de Santa Catarina, Fundação Jorge Duprat Figueiredo de Segurança \\ e Medicina do Trabalho, Florianópolis, SC, Brasil. \\ ${ }^{2}$ Curso Técnico de Meteorologia, Instituto Federal de Santa Catarina, \\ Florianópolis, SC, Brasil. \\ ${ }^{3}$ Escritório Regional de Campinas, Fundação Jorge Duprat Figueiredo \\ de Segurança e Medicina do Trabalho, Campinas, SP, Brasil. \\ ${ }^{4}$ Departamento de Ciências da Saúde, Universidade Federal dos Vales do Jequitinhonha \\ e Mucuri, Diamantina, MG, Brasil.
}

Recebido: 1/7/2015 - Aceito: 23/11/2015

\begin{abstract}
Resumo
As ondas de calor impactam setores socioeconômicos em diversas regiões do mundo. Dentro deste tema, o efeito do calor à saúde pública é um dos mais estudados, especialmente no que tange ao aumento da taxa de mortalidade humana. Procurando dar subsídios para possíveis ações mitigadoras no Brasil, o presente estudo propõe apresentar as características de frequência, duração, abrangência espacial e intensidade das ondas de calor. Utilizou-se dados de temperatura máxima diária $\left(T_{\max }\right)$ da série histórica (1961-2014) de 265 estações meteorológicas do Instituto Nacional de Meteorologia (INMET), separadas em três áreas do Brasil. Primeiramente, selecionou-se os eventos que, durante três ou mais dias, pelo menos a metade das observações na área apresentaram $T_{\max }$ superando a média de $T_{\max }$ mais um desvio padrão. Posteriormente, a média espacial e temporal da $T_{\max }$ foi definida como intensidade, Int. Por fim, os eventos com valores superiores ao terceiro quartil de Int foram definidos como ondas de calor. Foram encontradas 11 ondas de calor na área 1 , 23 na área 2 e 31 na área 3, a maioria na primavera e/ou verão. Nosso principal achado mostra que o número de ondas de calor tem aumentado ao longo das últimas cinco décadas, sendo a maioria das ocorrências concentradas após os anos 2000, na atual fase fria da Oscilação Decadal do Pacífico (ODP). Também nesse período, foram verificadas as ondas de calor mais duradouras e intensas. As conclusões deste trabalho sobre frequência, duração e intensidade das ondas de calor aparecem como forte indicativo de que as mudanças climáticas globais têm influenciado o campo de temperatura no Brasil.
\end{abstract}

Palavras-chave: onda de calor; mudança climática; saúde pública.

\section{Frequency, Duration, Spatial Coverage, and Intensity of Heat Waves in Brazil}

\begin{abstract}
Heat waves impact the socio-economic sectors of many world regions. At health public, for example, there are impacts on mortality rate. This study proposes to show the frequency, duration, spatial coverage, and intensity of heat waves. It was used daily maximum temperature $\left(T_{\max }\right)$ (1961-2014) observed by 265 meteorological stations separated by three areas of Brazil. At first, it was identified the events which, during three or more days, at least $50 \%$ of observations in the area had $T_{\max }$ greater than mean $T_{\max }$ plus one standard deviation. After, for each event, it was calculated the spatial and temporal mean of $T_{\max }$, which we called of intensity. Lastly, the events with intensity in upper quartile were called heat waves. The results showed 11 heat waves in area 1,23 in area 2, and 31 in area 3, the most of them in spring and summer.
\end{abstract}

Autor de correspondência: Daniel Pires Bitencourt, daniel.bitencourt@pq.cnpq.br. 
Our main finding shows an increase of heat waves during last five decades. In addition, the most of heat waves, being also the more intense and longer, occurred after 2000, during Pacific Decadal Oscillation (PDO) cool phase. Those findings appear how strong indications for actions of the climate changes on behavior of heat waves in Brazil.

Keywords: heat wave; climate change; health public.

\section{Introdução}

Em muitas regiões do mundo, o impacto do calor é notório em vários segmentos da sociedade. Períodos prolongados com temperaturas extremas são altamente prejudiciais à produtividade agrícola e pecuária. Geralmente, as ondas de calor também provocam aumentos significativos da demanda de consumo de energia elétrica. Entretanto, os impactos mais abordados na literatura são os prejuízos à saúde pública. Diversos autores associam a ocorrência de elevadas temperaturas com a procura por tratamentos médicos específicos ou emergências hospitalares (Hess et al., 2014; Tasian et al., 2014). No entanto, o principal aspecto abordado é o aumento das taxas de mortalidade humana (Semenza et al., 1996; Cont et al., 2005; Ostro et al., 2009; Hoshiko et al., 2010; Huang et al., 2010; Gasparrini e Armstrong, 2011; Lim et al., 2012; Jongsik e Kim, 2012; Monteiro et al., 2012).

Segundo Laaidi et al. (2012), o risco de morte por exposição a temperaturas altas é maior nas áreas urbanas devido ao efeito das chamadas ilhas de calor. Costa et al. (2010) encontraram diferenças significativas de temperatura entre áreas cobertas por vegetação e pavimentação na área urbana de Ilha Solteira, no estado de São Paulo (SP). Também em SP, Lima et al. (2013) investigaram a variação da temperatura do ar em áreas de mangue impactadas e conservadas. Os autores encontraram maior amplitude térmica, com máxima mais alta, nas áreas de mangue não conservadas. Segundo Jenerette et al. (2006), em ambientes urbanos são bastante claras as associações entre a temperatura do ar e as condições específicas dos bairros, tais como a economia, a densidade populacional e a taxa da área coberta por vegetação. Sabe-se, ainda, que as pessoas mais suscetíveis às ondas de calor são as crianças, os idosos e os adultos não saudáveis. Além disso, o potencial de risco é ainda maior para as populações de maior vulnerabilidade social. Harlan et al. (2006) mostraram que os grupos socioeconômicos mais baixos e os de minoria étnica da cidade de Phoenix, EUA, são mais propensos a viver em bairros quentes com maior exposição ao estresse térmico. Por outro lado, a queda da taxa de mortalidade percebida na onda de calor de 2006 pode estar associada à diminuição da vulnerabilidade das pessoas a este tipo de evento (Tan, 2008). Petkova et al. (2014) analisaram dados de Nova Yorque desde 1900 e também perceberam redução do risco de mortalidade devido ao calor a partir da segunda metade do século XX, indicando uma melhor adaptação ao calor por parte da população nas últimas décadas. Em outro estudo recente de Todd e Valleron (2015), através da análise espaço-temporal da relação mortalidade-temperatura, os resul- tados indicam alguma adaptação humana às mudanças climáticas e efeitos do calor na França. Possivelmente, os programas de prevenção e de informação pública sobre impactos do calor à saúde, além do desenvolvimento tecnológico aplicado a atenuação das condições extremas, sejam algumas das principais medidas mitigadoras para minimização dos efeitos do calor extremo sobre a população. Além dessas, os efeitos do calor são minimizados com a implantação de sistemas de alerta de saúde contra o calor, o uso de equipamentos de ar condicionado, execução de projetos de edificações mais ventiladas e acesso mais facilitado à água potável.

Apesar de algumas indicações, em geral bastante pontuais, de adaptação humana ao calor, as perspectivas globais de agravo das condições térmicas extremas apresentam-se cada vez mais claras. De acordo com o Painel Intergovernamental de Mudanças Climáticas (Intergovernmental Panel on Climate Change - IPCC) (IPCC, 2007), em muitas áreas do globo, com o advento das mudanças climáticas, dias e noites quentes tem sido mais intensos e frequentes. As perspectivas para o futuro são de manutenção destas tendências com sérios riscos à população mundial. Segundo o IPCC (2007), o recente período de 1995 a 2006 apresentou a maior temperatura média global desde 1850. O relatório The Global Climate 2001-2010, A Decade of Climate Extremes apresentou uma avaliação das condições extremas de tempo com base em dados de uma década e identificou no Brasil, entre janeiro e março de 2006, a ocorrência de uma das ondas de calor mais significantes do mundo. Meehl e Tebaldi (2004) preveem através de estudos numéricos que as ondas de calor na Europa e América do Norte serão mais frequentes, mais intensas e de maior duração na segunda metade do século XXI.

Dependendo do tipo de atividade e das condições de trabalho oferecidas aos trabalhadores de áreas externas, o calor extremo pode apresentar alto risco à saúde ocupacional, inclusive levando a morte. Bitencourt et al. (2012) encontraram relações do calor com mortes de cortadores de cana-de-açúcar no Estado de SP, onde trabalhadores desenvolviam atividades exaustivas com precárias condições de trabalho. Kjellstrom e McMichael (2013) ressaltaram sobre a necessidade de se identificar estratégias eficazes para reduzir as ameaças das mudanças climáticas, que incluem as ondas de calor, cada vez mais prováveis para a saúde e bem-estar humano. Iniciativas específicas, como a realização de estudos de campo, têm sido conduzidas para minimizar os efeitos danosos das mudanças climáticas à saúde e produtividade ocupacional (Kjellstrom et al., 2009). Entretanto, entende-se que qualquer ação estratégica em termos de políticas públicas com este fim requer estudos prévios 
que indiquem a situação vivida nas últimas décadas, assim como as perspectivas futuras em termos de tendência. Para o caso de extremos de calor no Brasil, alguns estudos têm sido conduzidos nos últimos anos, especialmente para a região Sul. Ribeiro e Nunes (2011) analisaram dados de uma estação meteorológica na cidade de Pelotas, Rio Grande do Sul (RS), e encontraram aumento da frequência de ondas de calor a partir da primeira década de 2000. Streck et al. (2011) constataram aumento da temperatura máxima a partir de meados da década de 1970 na cidade de Santa Maria, RS, e associaram este aumento com a fase quente da Oscilação Decadal do Pacífico (ODP). Com dados de toda a região Sul do Brasil, Marengo e Camargo (2008) encontraram associações dos eventos de El Niño com aquecimento e aumento da frequência de extremos de temperatura nas décadas de 1980 e 1990. Firpo et al. (2012) analisaram ondas de frio e calor utilizando 13 estações meteorológicas do RS e constataram que nos anos de El Niño ocorrem um número maior de ondas de calor. Cerne e Vera (2011) ressaltaram sobre o importante papel da Zona de Convergência do Atlântico Sul (ZCAS) na variabilidade intrasazonal das ondas de calor da região subtropical da América do Sul. Estudando séries de dados no período de 1961-2008 do estado do Ceará (CE), no Nordeste do Brasil, Andrade et al. (2012) encontraram aumento sistemático das temperaturas máxima e mínima especialmente a partir da década de 1990.

Todos estes estudos têm dado suporte para o aumento do conhecimento em termos de comportamento dos extremos de temperatura. Entretanto, não se encontra na literatura trabalhos que analisem estes extremos abrangendo todo o Brasil, embora atualmente exista considerável dis- ponibilidade de dados em todo o país. Uma macro análise deste tipo possui potencial para gerar subsídios para futuras ações mitigadoras. Diante do exposto, propõe-se aqui identificar e analisar a frequência, duração, abrangência espacial e intensidade das ondas de calor em três grandes áreas do Brasil, duas ao norte e uma ao sul de $15^{\circ} \mathrm{S}$ de latitude. Procura-se identificar tendências para as ondas de calor no período de 1961-2014 e associações destas tendências com variabilidades climáticas globais. Além disso, são avaliadas diferenças de comportamento entre as áreas analisadas.

\section{Materiais e Método}

Neste trabalho foram utilizados dados históricos de temperatura máxima do ar $\left(T_{\max }\right)$, observada pela rede de estações meteorológicas convencionais do Instituto Nacional de Meteorologia (INMET), disponíveis em http://www.inmet.gov.br/projetos/rede/pesquisa/. Em todo o Brasil, são 265 estações meteorológicas de superfície, as quais possuem maior densidade espacial nas regiões Sul, Sudeste e Nordeste (Fig. 1a). A série de dados contempla o período de 01/01/1961 a 30/10/2014, totalizando 19661 dias de observação. Os períodos com falta de dados não foram preenchidos. Em geral, estes períodos ocorrem em momentos diferentes em cada estação meteorológica, representando 10 a 40\% de toda a série histórica (Fig. 1a). Em algumas áreas do Brasil, como no centro da região CentroOeste, há estações com um percentual de falta de dados maior, entre 70 e $80 \%$.

Utilizando o limite latitudinal de $15^{\circ} \mathrm{S}$, separou-se uma porção do domínio brasileiro que em geral é influenciada por sistemas meteorológicos transientes de outra parte influenciada pela climatologia tropical. Esta região
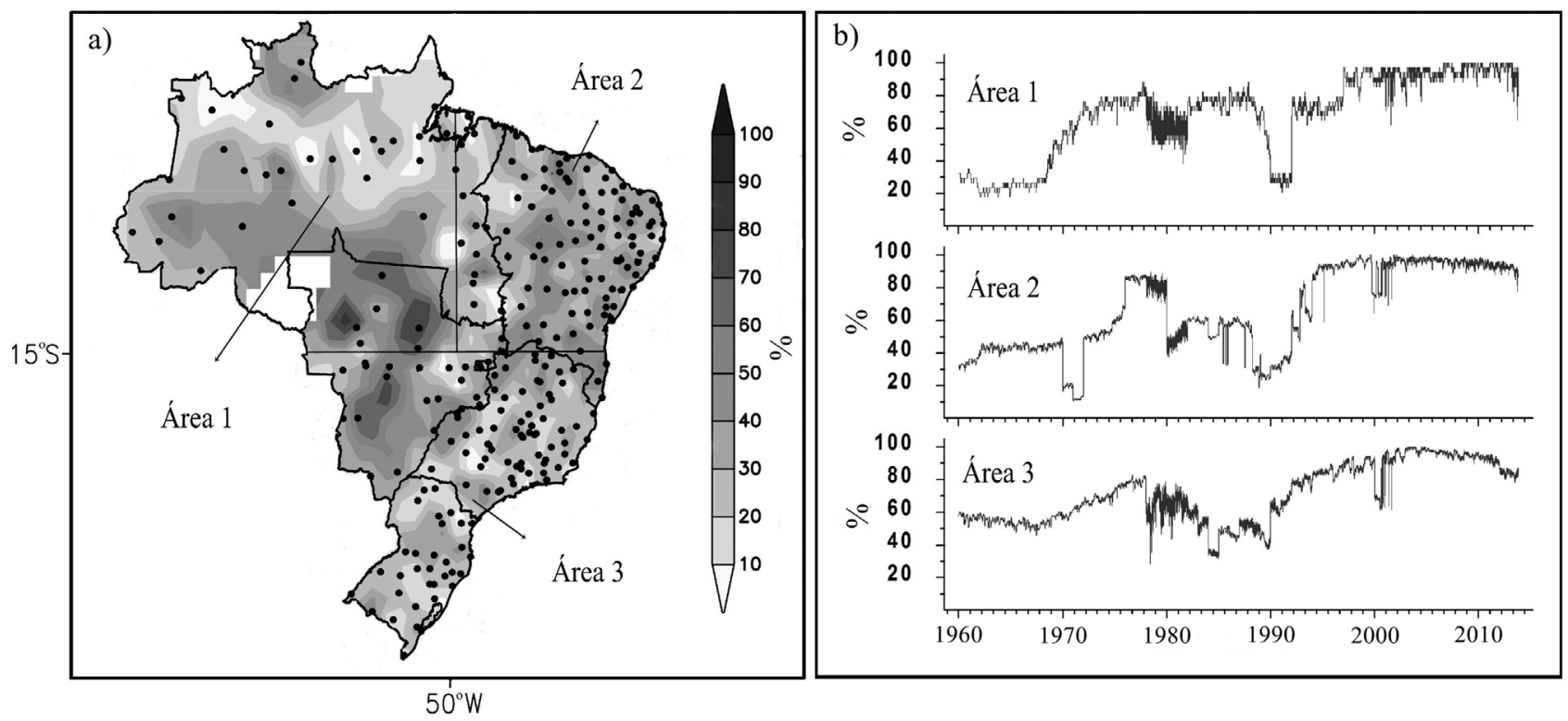

Figura 1 - (a) Localização (•) das 265 estações meteorológicas do INMET e percentual (\%) de dados inexistentes em relação ao total de 19661 dias da série. As linhas retas nas marcas de $15^{\circ} \mathrm{S}$ e $50^{\circ} \mathrm{W}$ limitam as áreas 1,2 e 3, conforme identificação no mapa. (b) Percentual (\%) diário (série de 01/01/1961 a 30/10/2014) de dados existentes em relação ao total de 34 estações meteorológicas na área 1, 107 na área 2 e 124 na área 3. 
tropical foi ainda subdividida em duas áreas com significativa diferença em termos de disponibilidade de dados meteorológicos. Dessa forma, a identificação das ondas de calor foi realizada separadamente para três áreas no Brasil (Fig. 1a). O domínio ao norte de $15^{\circ} \mathrm{S}$ de latitude e oeste de $50^{\circ} \mathrm{W}$ de longitude é definido como área 1 e é representado por 34 estações meteorológicas. A área 2 contempla o domínio ao norte de $15^{\circ} \mathrm{S}$ de latitude e leste de $50^{\circ} \mathrm{W}$ de longitude, com 107 estações meteorológicas. A área 3 é representada por 124 estações meteorológicas e contempla todo o domínio ao sul de $15^{\circ} \mathrm{S}$ de latitude. Este número total de estações meteorológicas em cada área não ficou disponível ao longo de toda a série histórica. Conforme gráficos apresentados na Fig. $1 \mathrm{~b}$ verifica-se aumento da disponibilidade de dados ao longo da série, nas áreas 1, $2 \mathrm{e}$ 3. Isto é devido, principalmente, ao menor número de estações meteorológicas existentes no início da série em relação à disponibilidade atual.

Para os propósitos do presente trabalho, a baixa disponibilidade de dados não inviabiliza a identificação das ondas de calor, desde que os dados estejam bem distribuídos espacialmente e representem a área em sua totalidade. Esta representação é fundamental porque a abrangência espacial faz parte do critério de identificação das ondas de calor. Por isso, com o auxílio dos gráficos apresentados na Fig. 1b e por meio de avaliação subjetiva (visualização da distribuição espacial dos dados - figura não mostrada), antes de serem aplicados os critérios de identificação das ondas de calor, foram desconsiderados alguns períodos da série de dados com baixa representatividade espacial da área. Os períodos excluídos foram de janeiro de 1991 a dezembro de 1992 na área 1, de janeiro a dezembro de 1972 e de abril de 1989 a dezembro de 1990 na área 2 e de janeiro a setembro de 2001 na área 3.

Robinson (2001) ressalta que uma das características das ondas de calor é a capacidade da temperatura afetar a maioria da população, o que requer alteração em suas atividades normais para evitar desconforto ou problemas de saúde. Outro aspecto abordado por Robinson (2001) é o desvio do parâmetro avaliado (nesse estudo, considera-se a $T_{\max }$ ) em relação aos valores médios. O afastamento das condições esperadas ou médias estipula uma padronização dos extremos entre várias localidades dentro de uma determinada área, independentemente das características de clima local de cada localidade. Radinovié e Curié (2012) contestam a recomendação do IPCC, reportada por $\mathrm{Cu}-$ basch et al. (2001), para definição das ondas de calor. Esta recomendação considera um período de cinco ou mais dias consecutivos com temperatura máxima diária excedendo $5{ }^{\circ} \mathrm{C}$ ou mais a temperatura máxima média, obtida do período de 1961 a 1990 (IPCC, 2001). Radinovié e Curié (2012) reforçam, portanto, a necessidade de identificar as ondas de calor com base em limites que consideram a soma do valor médio mais um ou dois valores de desvio padrão. Conforme detalhes mostrados a seguir, neste estudo serão adotados estes limites para a identificação e/ou caracterização das ondas de calor, além de também considerar um limite mínimo para duração e abrangência espacial.

Primeiramente, calcula-se a média diária $(d)$ da temperatura máxima para cada estação meteorológica $(k)$, utilizando a Eq. (1).

$$
\bar{T}_{\max }(k, d)=\frac{1}{N(k, d)} \sum_{i=1961}^{2014} T_{\max (i)}(k, d)
$$

onde $k=1,2,3, \ldots, 265$ (número de estações meteorológicas), $d=1,2,3, \ldots, 365$ (dias do ano) e $N(k, d)$ é o número de anos com existência de dados, para a estação $k \mathrm{e}$ dia $d$.

O desvio padrão $(\sigma)$ diário $(d)$ para cada estação meteorológica $(k)$ é dado pela Eq. (2).

$$
\sigma(k, d)=\frac{\sqrt{\sum_{i=1961}^{2014}\left(T_{\max (i)}(k, d)-\bar{T}_{\max }(k, d)\right)^{2}}}{N(k, d)-1}
$$

Portanto, há 365 valores de temperatura máxima média $\left(\bar{T}_{\max }\right)$ e de desvio padrão $(\sigma)$ para cada uma das 265 estações meteorológicas.

Posteriormente, foi identificado na série de dados os eventos de três ou mais dias consecutivos com registro de $T_{\max } \geq \bar{T}_{\max }+\sigma$ (temperatura máxima maior ou igual que a média da temperatura máxima acrescida de 1 (um) desvio padrão) em pelo menos $50 \%$ das estações meteorológicas da área. Na área 1, 42 eventos obedeceram este critério, na área 2 foram 91 eventos e na área 3 foram 122 eventos. Para cada um destes eventos, calculou-se a média diária de $T_{\max }$ entre as estações meteorológicas (mínimo de $50 \%$ das estações) com registro de $T_{\max } \geq \bar{T}_{\max }+\sigma$. Depois, calculou-se o valor médio entre os dias (mínimo 3 dias) do evento. $\mathrm{O}$ parâmetro, dado pela média de $T_{\max }$ entre as estações meteorológicas e entre os dias do evento, é definido como intensidade (Int). Os eventos com Int acima do limite superior ao terceiro quartil da série são classificados como onda de calor. Este último procedimento foi realizado com intuito de selecionar apenas os eventos extremamente impactantes em termos de calor que, segundo Robinson (2001), é uma característica inerente de uma onda de calor. Com este filtro, são eliminados os eventos que, embora obedeçam aos critérios de "mínimo de três dias com pelo menos 50\% das observações registrando $T_{\max } \geq \bar{T}_{\text {max }}+\sigma$ ", não são caracterizados por períodos efetivamente quentes e, portanto, não possuem potencial para impactar a sociedade e os setores socioeconômicos de forma mais ampla. Para a área 1, o limite superior ao terceiro quartil é $I n t=35,83^{\circ} \mathrm{C}$, para a área 2 é $I n t=34,95{ }^{\circ} \mathrm{C}$ e para a área 3 é $I n t=34,02^{\circ} \mathrm{C}$. Após a aplicação de todos estes critérios, identificou-se 11 ondas de calor na área 1, 23 ondas de calor na área 2 e 31 ondas de calor na área 3.

O esquema mostrado na Fig. 2 ilustra duas ondas de calor hipotéticas (dados não reais), com o objetivo de deta- 


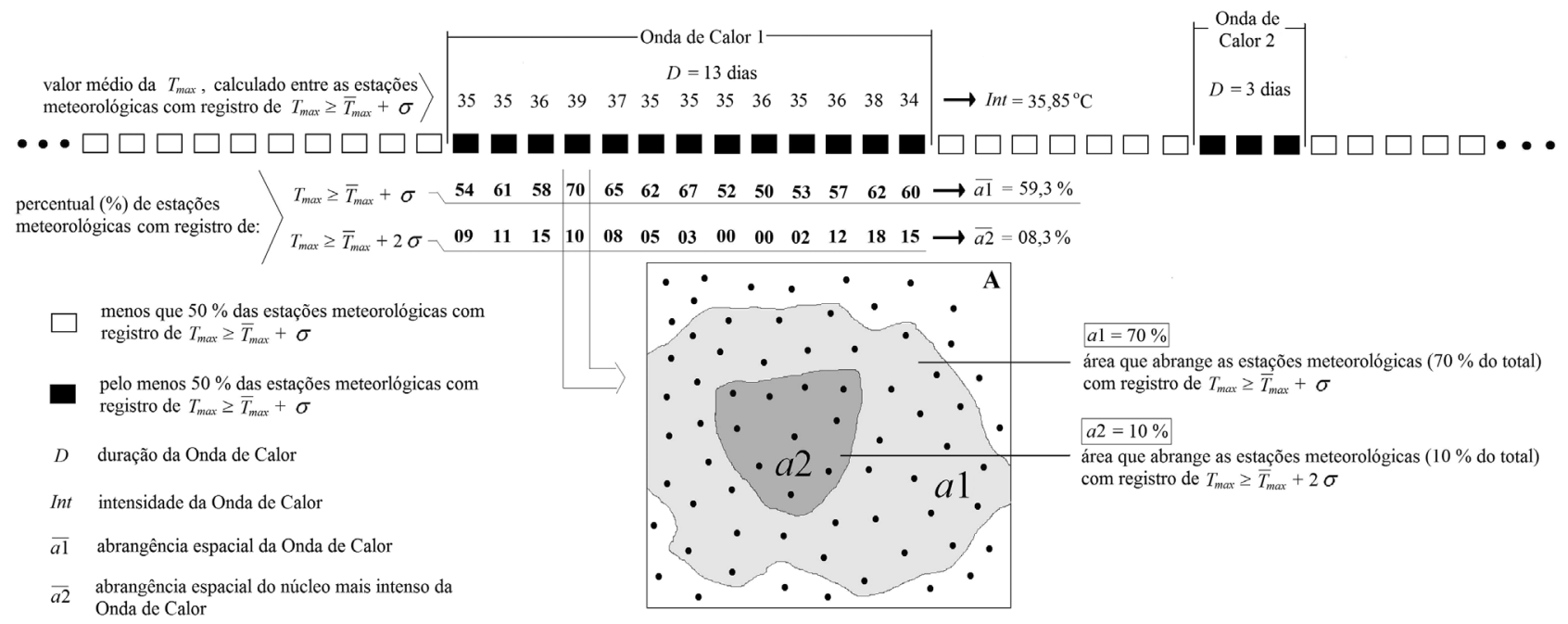

Figura 2 - Exemplo ilustrativo de duas ondas de calor e da área $\mathbf{A}$, representante de uma situação hipotética do quarto dia da Onda de Calor número 1. Para esta situação, $70 \%$ das estações meteorológicas estariam registrando $T_{\max } \geq \bar{T}_{\max }+\sigma$ e $10 \%$ das estações meteorológicas estariam registrando $T_{\max } \geq \bar{T}_{\max }+2 \sigma$. O trecho da série histórica, as duas ondas de calor e a área apresentada não representam situações reais, são ilustrações hipotéticas.

lhar o método de identificação aqui adotado. Nesta ilustração, a onda de calor 1 possui 13 dias consecutivos com no mínimo $50 \%$ das estações meteorológicas registrando $T_{\max } \geq \bar{T}_{\max }+\sigma$. A onda de calor 2 possui 3 dias (duração mínima, segundo o critério) consecutivos atendendo os mesmos critérios. Considerando esta representação hipotética, a onda de calor 1 apresenta intensidade $\operatorname{Int}=35,85^{\circ} \mathrm{C}$, duração $D=13$ dias, abrangência espacial $\bar{a}_{1}=59,3 \%$ e abrangência espacial do núcleo mais intenso $\bar{a}_{2}=8,3 \%$. O esquema da Fig. 2 mostra ainda o que seriam as representações espaciais $a_{1}$ e $a_{2}$ no quarto dia da onda de calor 1 . Na prática, $\bar{a}_{1} \geq 50 \%$ e $\bar{a}_{2} \geq 0 \%$, sendo os respectivos cálculos dados por $\quad \bar{a}_{1}=D^{-1} \sum_{\text {dia }=1}^{\text {dia }=D} a_{1(\text { dia })} \quad \mathrm{e}$ $\bar{a}_{2}=D^{-1} \sum_{\text {dia }=1}^{\text {dia }=D} a_{2(d i a)}$.

\section{Resultados e Discussões}

\subsection{Frequência mensal e tendência no período 1961-2014}

$\mathrm{Na}$ área 1, as 11 ondas de calor ocorreram somente no trimestre agosto-setembro-outubro, sendo mais da metade das ocorrências em setembro (Fig. 3a). Na área 2, as 23 ondas de calor ocorreram entre setembro e março, com pico máximo de ocorrência (aproximadamente $30 \%$ ) em janeiro (Fig. 3b). Os meses de setembro e outubro contabilizaram mais de 30\% das ocorrências de ondas de calor na área 2 . $\mathrm{Na}$ área 3, as 31 ondas de calor ocorreram entre setembro e fevereiro, sendo outubro o mês de maior ocorrência (aproximadamente 40\%) (Fig. 3c). Portanto, excetuando frequências relativamente baixas em agosto na área $1 \mathrm{e} e \mathrm{em}$ março na área 2, verifica-se que as ondas de calor ocorrem nos meses de primavera e/ou verão. No domínio ao sul de $15^{\circ} \mathrm{S}$ de latitude (área 3), onde as condições de tempo são dominadas por sistemas meteorológicos transientes e as estações do ano são bem definidas em termos de temperatura, a primavera concentra as maiores frequências de ondas de calor em relação ao verão (Fig. 3c).

Marengo e Valverde (2007) mostraram que elevações da temperatura podem estar associadas a fatores naturais, tais como anomalias de Temperatura da Superfície do Mar (TSM) no Oceano Atlântico e Oceano Pacífico (El Niño Oscilação Sul - ENOS) ou a fatores antropológicos, tais como queima de combustível fóssil, industrialização, mudanças no uso da terra e subsequentes queimadas. No processo de aquecimento, há ainda que se considerar a ODP. Utilizando quase um século de dados, Streck et al. (2011) estudaram a variabilidade interdecadal das temperaturas mínima e máxima na cidade de Santa Maria, RS, e encontraram decréscimo dessas temperaturas durante a fase fria da ODP de 1947-1976 e aumento da temperatura máxima na fase quente, de 1977-1998.

Neste trabalho, a tendência do número de ondas de calor é avaliada sob a consideração das fases quente e fria da ODP (Mantua et al., 1997) e da ocorrência dos eventos de El Niño e La Niña, obtidos por Trenberth (1997) através do Índice Oceânico Niño (ION). A série de dados aqui analisada (Fig. 4) nesse trabalho coincide com os últimos 14 anos da fase fria da ODP finalizada em 1976, com toda a fase quente de 1977-1998 (Mantua e Hare, 2002) e com os primeiros 16 anos da nova fase fria iniciada em 1999. Verifica-se que durante a fase fria da ODP finalizada em 1976 ocorreram ondas de calor somente na área 3. Conforme mostrado na Fig. 4c, foram 3 ondas de calor ocorridas entre outubro e dezembro de 1963. Durante a fase quente de 1977-1998 foi verificado uma onda de calor na área 1 (em 1995) (Fig. 4a) e 5 ondas de calor na área 2 (3 nos anos de 1981, 1983 e 1987 e duas em 1997) (Fig. 4b). Na área 3, durante a fase quente da ODP de 1977-1998, foram identificadas 12 ondas de calor, nos anos de 1985, 1987, 1988, 



Figura 3 - Frequência (\%) mensal de ondas
na área 2 e (c) 31 ondas de calor na área 3.

1990, 1994, 1995, 1997 e 1998 (Fig. 4c). Portanto, entre o período considerado da fase fria de 1947-1976 e a fase quente de 1977-1998, percebe-se um claro aumento do número de ondas de calor nas áreas 2 e 3 . Streck et al. (2011), com dados da cidade de Santa Maria, RS, observaram aumento de temperatura durante este mesmo período e associaram este resultado a mudança da fase fria para a fase quente da ODP.

Contudo, nos dados aqui analisados, durante os primeiros 16 anos da nova fase fria da ODP, iniciada em 1999, o número de ondas de calor apresentou um aumento ainda mais acentuado nas três áreas. Na área 1 ocorreram 10 ondas de calor (em 2002, 2005, 2009, 2010 e 2011), na área 2 ocorreram 18 ondas de calor (em 2003, 2005, 2006, 2007, 2008, 2009, 2010, 2012 e 2013) e, na área 3, são 16 novas ondas de calor (em 1999, 2000, 2002, 2003, 2004, 2006, 2007, 2008, 2010, 2012 e 2014). Portanto, no domínio ao norte de $15^{\circ} \mathrm{S}$ (áreas 1 e 2), somente nos primeiros 16 anos da nova fase fria já ocorreram significativamente mais ondas de calor do que todo o restante da série que inclui parte de uma fase fria e a integralidade de uma fase quente da ODP. No domínio ao sul de $15^{\circ} \mathrm{S}$ (área 3) mais da metade das ondas de calor identificadas na série de 19612014 ocorreram nos primeiros 16 anos da fase fria da ODP iniciada em 1999. As linhas de tendência, apresentadas na Fig. 4, mostram claramente o incremento contínuo da fre- quência de ondas de calor nas áreas 1, 2 e 3. Este resultado chama atenção por seu caráter singular, não associado a padrões de variabilidades climáticas conhecidas, podendo desta forma estar associado com mudanças climáticas globais. Em paralelo a esta constatação, deve-se também considerar a possibilidade, embora baixa, de nos próximos anos da atual fase fria da ODP o número de ondas de calor passar a diminuir. Além disso, é prudente também considerar que a elevação das temperaturas seja uma consequência de possíveis efeitos de urbanização nos arredores das estações meteorológicas. Por outro lado, com a metodologia de identificação das ondas de calor proposta neste estudo, o efeito de urbanização somente causaria algum impacto na análise caso esta situação tivesse sido verificada em muitas localizações.

No período de 1961 a 2014, muitos anos de El Niño ou de La Niña não apresentaram ondas de calor. Um exemplo disso é o período entre 1968 e 1977, que em todos os anos apresentaram El Niño ou La Niña, porém, sem registro de ondas de calor em qualquer uma das áreas aqui estudada. Contudo, quando ocorreram ondas de calor na área 1, 9 casos foram em anos de El Niño e apenas 2 em anos de neutralidade climática. $\mathrm{Na}$ área 2, 11 ondas de calor foram com situação climática de El Niño, 10 de neutralidade e apenas 2 de La Niña. No domínio ao sul de $15^{\circ} \mathrm{S}$ (área 3), 15 casos de ondas de calor ocorreram com El Niño, 6 com 


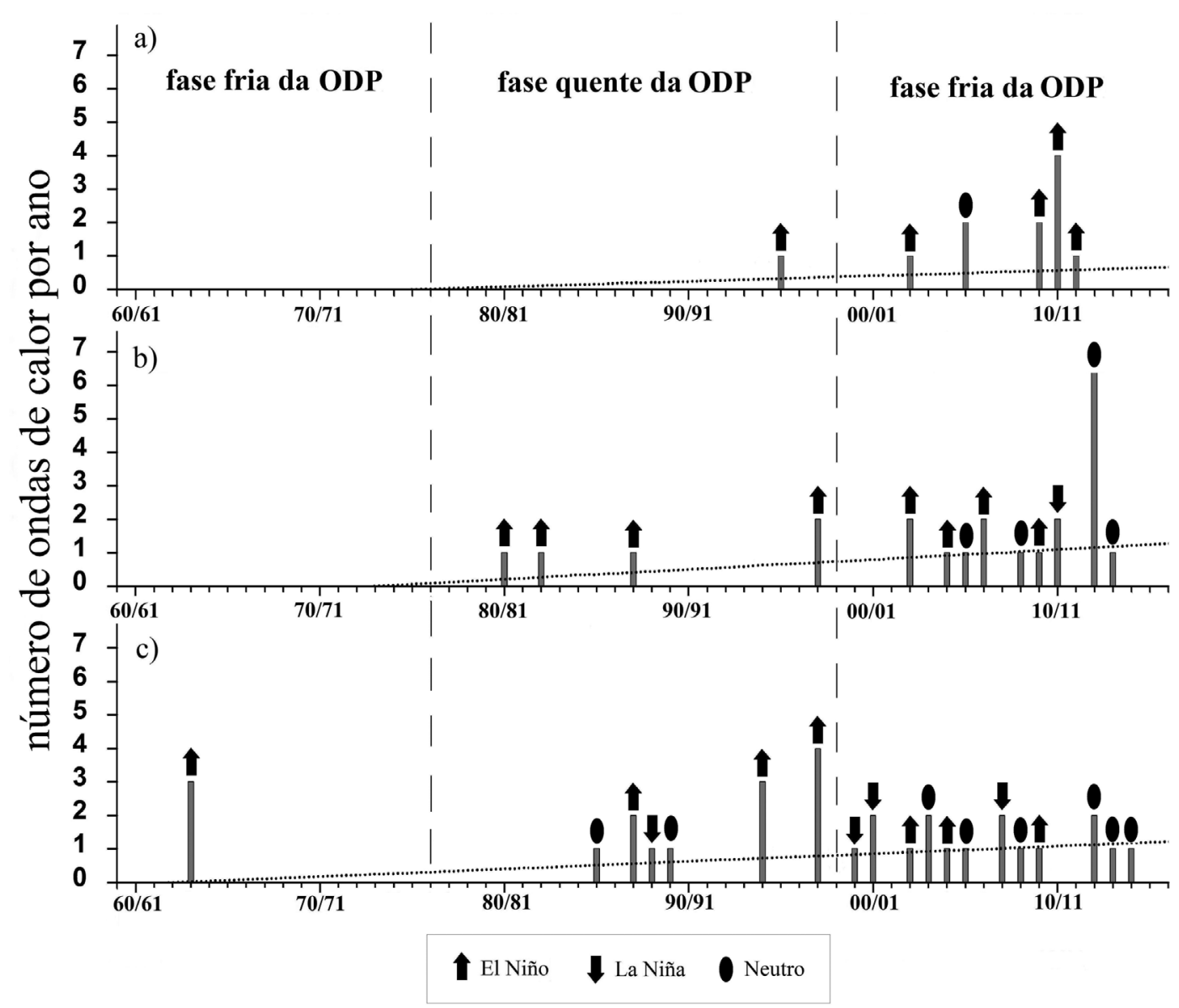

Figura 4 - Número de ondas de calor (barras cinza) a cada 12 meses nas áreas (a) 1, (b) 2 e (c) 3, identificadas entre agosto de um ano e julho do próximo. A série de dados inicia em 1961/1962 (a partir de 01 de janeiro de 1961) e finaliza em 2014/2015 (até 30 de outubro de 2014). A linha pontilhada representa a tendência definida por meio de modelo linear simples. Os símbolos definidos na caixa da base da figura indicam o tipo de ENOS na ocasião de ocorrência das ondas de calor. O tipo de ENOS obedece a classificação definida pelos valores trimestrais do Índice Oceânico Niño (ION) (Trenberth, 1997), disponível em http://www.cpc.noaa.gov/products/analysis_monitoring/ensostuff/ensoyears.shtml. Os limites temporais das fases fria e quente da Oscilação Decadal do Pacífico (ODP) (Mantua e Hare, 2002) são indicados pelas linhas verticais tracejadas e identificadas no topo da figura.

La Niña e 10 em situação de neutralidade climática. Portanto, principalmente para o domínio ao norte de $15{ }^{\circ} \mathrm{S}$, parece existir uma preferência de ocorrência de ondas de calor em anos de El Niño ou neutros. $\mathrm{Na}$ área 3, ocorreram mais ondas de calor (6 casos) com situação de La Niña em relação as demais áreas analisadas. Este resultado pode ser devido à grande extensão considerada para a área 3, abrangendo as regiões Sul e Sudeste e, pelo menos, a metade sul da região Centro-Oeste do Brasil. Marengo e Camargo (2008), analisaram 27 estações meteorológicas da região Sul e constataram que a presença de eventos de El Niño com maior intensidade e frequência durante as décadas de 1980 e 1990 contribui para o aquecimento verificado nestes 20 anos. Isto também explica o aumento acentuado de extremos de temperatura encontrado por Marengo e Camargo (2008) entre 1980-2000, em comparação com o período relativamente mais frio ocorrido entre 1960-1980. Com dados apenas do RS, Firpo et al. (2012) também constataram que nos anos de El Niño ocorre um número maior de ondas de calor. Por outro lado, Kayano e San- sígolo (2009) analisaram dados de 1913-2006 de cinco estações meteorológicas do RS e concluíram que a variabilidade das temperaturas neste estado é mais relacionada com a variabilidade da TSM no Oceano Atlântico do que no Oceano Pacífico.

\subsection{Duração, abrangência espacial e intensidade}

A duração $(D)$ das ondas de calor não difere muito entre as três áreas (Fig. 5). Os períodos médios de duração nas áreas 1, 2 e 3 são, respectivamente, 4,5, 4,6 e 4,7 dias. Na área 1 , a onda de calor de maior duração persistiu durante 8 dias. Na área 2 o maior período de onda de calor durou 12 dias e na área 3 foram 10 dias. Entretanto, em termos de duração deve-se considerar que os períodos apresentados na Fig. 5 obedecem estritamente aos critérios de identificação estipulados neste trabalho. E, seguindo estes critérios, ocorre algumas vezes de duas ondas de calor serem separadas por um pequeno intervalo de dias. $\mathrm{Na}$ área 1 ocorrem três casos em que duas ondas de calor são separadas por períodos de 5 ou 10 dias. Na área 2 ocorre um 


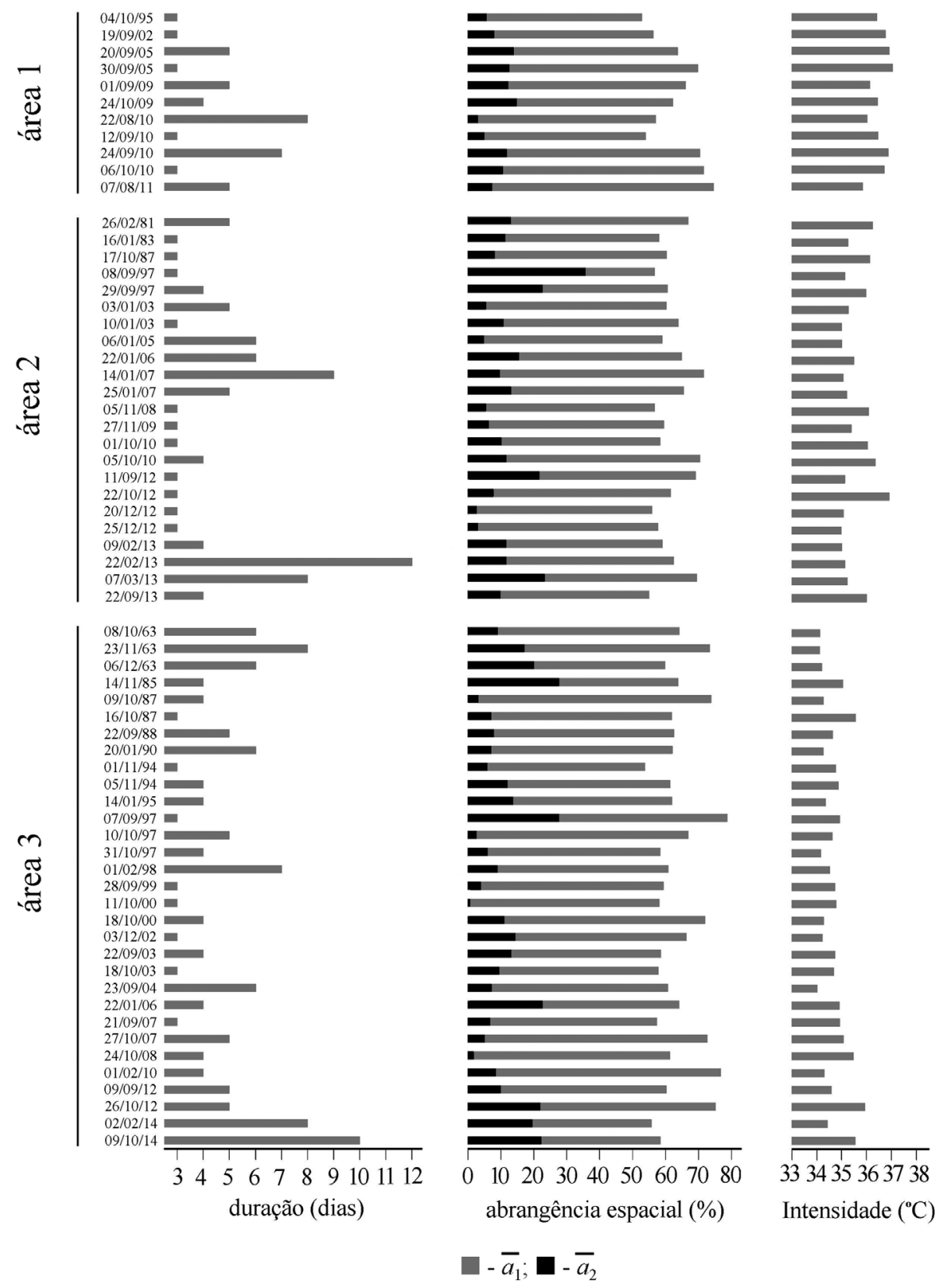

Figura 5 - Duração $(D)$, abrangência espacial e intensidade (Int) das ondas de calor identificadas nas áreas 1, 2 e 3 . A data de início da onda de calor é mostrada a esquerda do gráfico de $D$.

caso de duas ondas de calor separadas por um período de 10 dias e mais quatro casos em que duas ondas de calor são separadas por intervalos de apenas 1, 2 ou 3 dias. Já na área 3 , em quatro ocasiões duas ondas de calor são separadas por intervalos de 1, 4 ou 5 dias. Isso implica no fato de que a sequência de duas ou até três ondas de calor apresentadas nos gráficos da Fig. 5 possam ser eventos forçados pela mesma condição climática vigente na área. Ou seja, uma sequência de duas ou três ondas de calor separadas por períodos relativamente curtos são, na verdade, parte de um único período extremamente quente. Como visto na seção anterior, o maior número de ondas de calor ocorre na nova fase fria da ODP iniciada em 1999. Na Fig. 5, percebe-se um resultado análogo com respeito a duração das ondas de calor, pois as mais duradouras concentram-se nos últimos 16 anos da série analisada. Por outro lado, em termos de abrangência espacial das ondas de calor, isso não é verificado.

Considerando as três áreas, a maioria das ondas de calor com duração superior a 5 dias possui abrangência espacial $\bar{a}_{1}$ superior a $60 \%$. Ou seja, em geral as ondas de calor mais duradouras são também as que atingem as maiores áreas. O inverso também é verificado, pois mais da metade das 24 ondas de calor com duração mínima de 3 
dias não possuem a abrangência espacial $\bar{a}_{1}$ muito significativa, com $\bar{a}_{1}<60 \%$. Entretanto, a onda de calor mais abrangente de todas, que ocorreu em setembro de 1997, na área 3, apresentou duração de apenas 3 dias. Em geral, a abrangência $\bar{a}_{1}$ (percentual das estações meteorológicas com $T_{\max } \geq \bar{T}_{\max }+\sigma$ ) não difere muito entre as três áreas. Nas áreas 1,2 e 3 , os valores médios de $\bar{a}_{1}$ são, respectivamente, $63,5,61,2$ e $63,8 \%$. A abrangência espacial do núcleo mais intenso da onda de calor $\left(\bar{a}_{2}\right)$, dada pela porcentagem de estações meteorológicas com $T_{\max } \geq \bar{T}_{\max }+2 \sigma$, em geral, acompanha a abrangência espacial $\bar{a}_{1}$. Ou seja, quanto maior a área representada por estações meteorológicas que registram $T_{\max } \geq \bar{T}_{\max }+\sigma$, maior também é a área representada por estações meteorológicas que registram $T_{\max } \geq \bar{T}_{\max }+2 \sigma$.

A intensidade média das ondas de calor é maior na área $1 \mathrm{com} \overline{\mathrm{Int}}=36,5{ }^{\circ} \mathrm{C}$, seguida pela área $2 \mathrm{com}$ $\overline{I n t}=35,5^{\circ} \mathrm{C}$ e pela área $3 \mathrm{com} \overline{I n t}=34,7{ }^{\circ} \mathrm{C}$. A onda de calor mais intensa $\left(\operatorname{Int}=37,05^{\circ} \mathrm{C}\right)$ da área 1 ocorreu entre 30 de setembro e 02 de outubro de 2005 . Esta onda de calor foi a mais intensa do Brasil e teve abrangência espacial $\bar{a}_{1}=69,8 \%$ e $\bar{a}_{2}=12,5 \%$ (Fig. 5). A onda de calor mais intensa $\left(\right.$ Int $\left.=36,91{ }^{\circ} \mathrm{C}\right)$ da área 2 ocorreu entre 22 e 24 de outubro de $2012 \operatorname{com} \bar{a}_{1}=61,5 \%$ e $\bar{a}_{2}=7,7 \%$. Na área 3 , a onda de calor mais intensa $\left(\right.$ Int $\left.=35,94^{\circ} \mathrm{C}\right)$ iniciou em 26 de outubro de 2012 e teve $D=5$ dias, $\bar{a}_{1}=75,1 \%$ e $\bar{a}_{2}=22 \%$. Portanto, entre essas ondas de calor mais intensas de cada área, esta última, na área 3, foi a mais duradoura e abrangente. Ressalta-se ainda que essas três ondas de calor ocorreram na fase fria da ODP iniciada em 1999.

Em duas ocasiões, entre 22 e 25 de janeiro de 2006 e entre 11 e 13 de setembro de 2012, as áreas 2 e 3 apresentam simultaneidade de ondas de calor. O primeiro caso ocorreu dentro do período citado no relatório The Global Climate 2001-2010, A Decade of Climate Extremes, como sendo o período de ocorrência de uma das ondas de calor mais significativas do mundo.

Com intuito de mostrar o comportamento pontual da $T_{\max }$ na ocasião das ondas de calor mais intensas de cada área, apresenta-se na Fig. 6 o dia mais quente ocorrido durante cada um destes eventos. Analisando esses dias individualmente, nota-se que a $\operatorname{Int}(d)$, dada pela média da $T_{\max }$ entre as estações meteorológicas que apresentaram $T_{\max } \geq \bar{T}_{\max }+\sigma$ no dia $d$, não difere muito entre as três áreas. Também se nota que os valores absolutos de $T_{\max }$ ficam próximos a $40{ }^{\circ} \mathrm{C}$ em vários pontos de qualquer uma das três áreas (Fig. 6). Na área 1, uma estação meteorológica registrou $T_{\max }=39,7{ }^{\circ} \mathrm{C}$ no sul do estado do Amazonas (AM) (Fig. 6a). Na área 2, os valores de $T_{\max } \geq 40^{\circ} \mathrm{C}$ foram observados por 9 estações meteorológicas, com extremo $\left(T_{\max }=41,7^{\circ} \mathrm{C}\right)$ registrado no leste do estado do Maranhão (MA). Na área 3, 11 estações meteorológicas observaram $T_{\max } \geq 40^{\circ} \mathrm{C}$, com valor extremo de $T_{\max }\left(42,6^{\circ} \mathrm{C}\right)$ registrado em dois pontos da área, um no nordeste e outro no leste do estado de Minas Gerais (MG). Este diagnóstico, retra- tando apenas um dia da onda de calor mais intensa em cada área, revela que a área 3 , com domínio ao sul de $15^{\circ} \mathrm{S}$, é a área que apresenta pontualmente as temperaturas mais críticas.

Também se nota na análise da Fig. 6 que há diferenças entre as três áreas quanto ao distanciamento da $T_{\max }$ acima da $\left(\bar{T}_{\max }+2 \sigma\right)$. Percebe-se que, além da área 3 ter apresentado uma abrangência espacial $\left(a_{1}\right)$ maior em relação as demais áreas, esta área também apresenta a abrangência espacial $a_{2}$, definida pela porcentagem de dados com $T_{\max } \geq \bar{T}_{\max }+2 \sigma$, com dimensões bem superiores que as áreas 1 e 2. Enquanto na área 3 a porcentagem de estações com $T_{\max } \geq \bar{T}_{\max }+2 \sigma$ foi de $43,5 \%$, nas áreas 1 e 2 foram, respectivamente, 21,9 e $5 \%$.

\section{Considerações Finais}

No presente trabalho, ondas de calor entre 1961 e 2014 foram identificadas por meio da utilização de extremos de temperatura máxima diária, considerando aspectos de duração (mínimo de 3 dias), abrangência espacial (mínimo de $50 \%$ da área) e intensidade (limite superior ao terceiro quartil) em três grandes áreas do Brasil, duas ao norte e uma ao sul de $15{ }^{\circ} \mathrm{S}$ de latitude. Neste período de pouco mais de cinco décadas, foram identificadas 11 ondas de calor na área de número 1 (aproximadamente a metade da região Centro-Oeste e quase toda a região Norte), 23 ondas de calor na área número 2 (pequena parte das regiões Norte e Centro-Oeste e quase toda região Nordeste) e 31 ondas de calor na área número 3 (pequena parte da região Nordeste, aproximadamente metade da região CentroOeste e as regiões Sudeste e Sul).

As principais constatações encontradas a respeito da frequência, duração, abrangência espacial e intensidade das ondas de calor foram as seguintes:

- Com exceção das baixas frequências de ocorrência registradas nos meses de agosto (área 1) e março (área 2), as ondas de calor no Brasil ocorrem somente na primavera e/ou verão. Os meses com maiores frequências de ocorrência são setembro na área 1, janeiro na área 2 e outubro na área 3.

- Em relação à fase fria da ODP finalizada em 1976, o número de ondas de calor aumentou durante a fase quente da ODP de 1977-1998. Posteriormente, durante os quase 16 anos da nova fase fria da ODP, iniciada em 1999, o número de ondas de calor aumentou ainda mais. Somente esses quase 16 anos da nova fase fria da ODP concentraram 10 das 11 ondas de calor registradas na área 1,18 das 23 ondas de calor registradas na área 2 e 16 das 31 ondas de calor registradas na área 3. Estes resultados apontam para efeitos provocados pelas mudanças climáticas globais, implicando numa frequência de ocorrência de ondas de calor significativamente maior a partir do ano 2000. Contudo, apesar das fortes evidências de aumento do número de ondas de calor durante a atual fase fria da ODP em relação às fases anteriores, é neces- 
a)

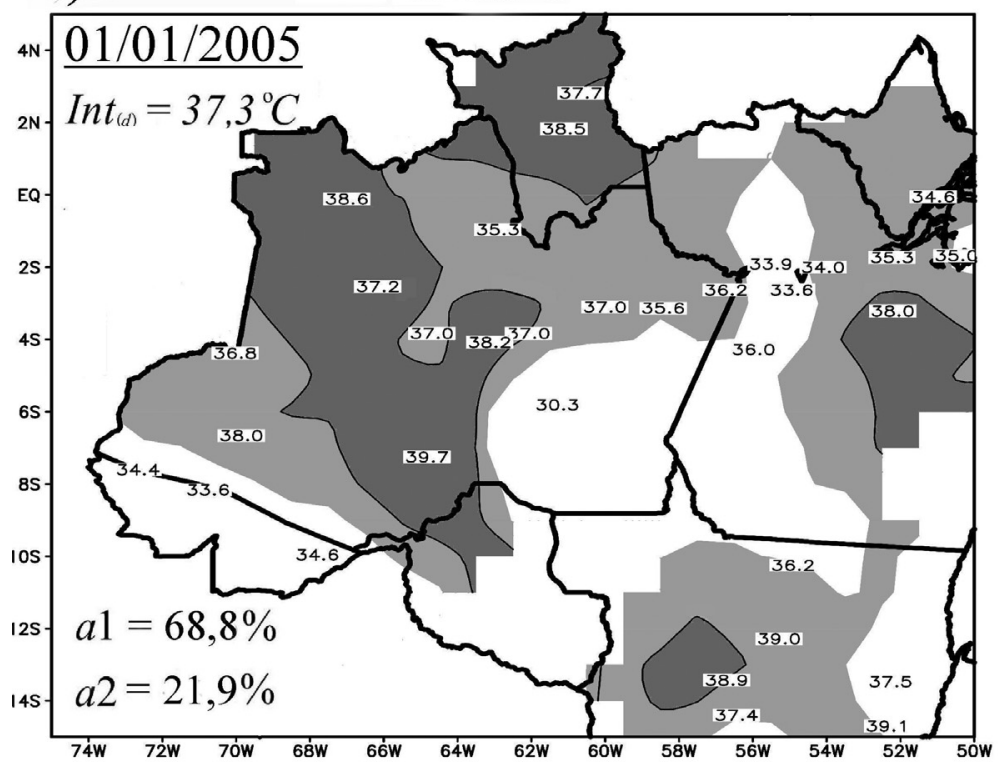

b) área 2

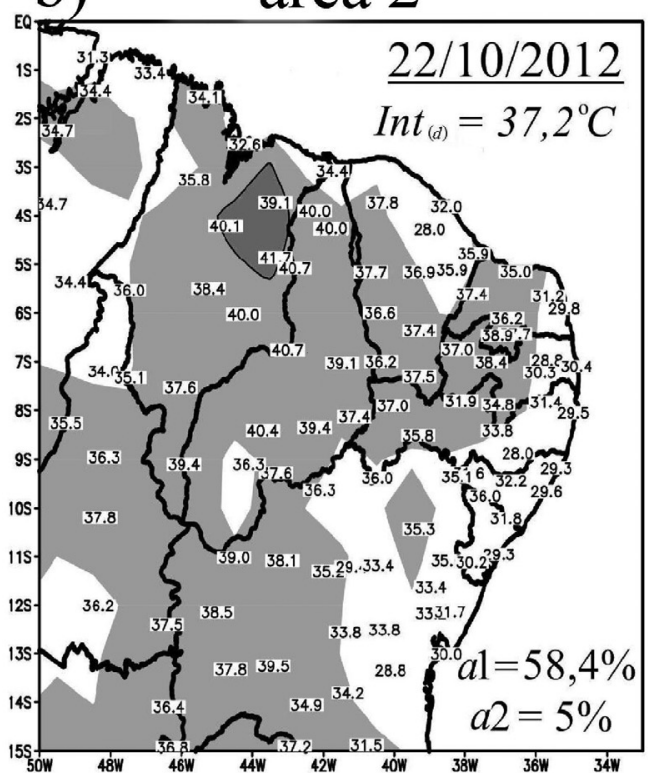

c) área 3

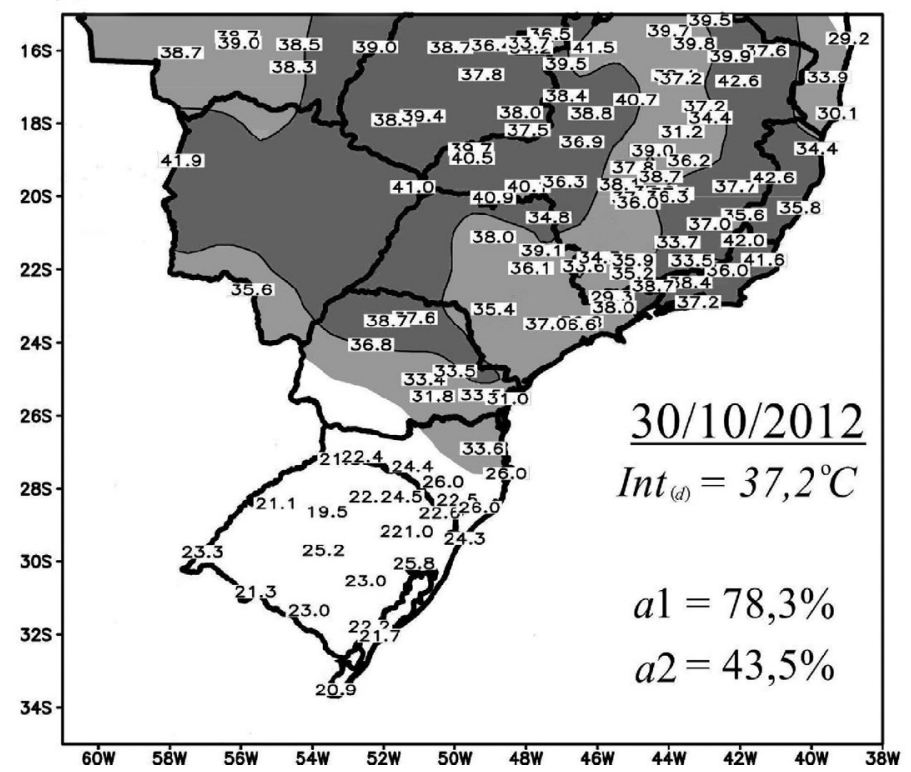

Figura 6 - Temperatura máxima $\left(T_{\max }\right)$ observada no dia de maior intensidade da onda de calor identificada (a) entre 30 de setembro e 02 de outubro de 2005 na área 1, (b) entre 22 e 24 de outubro de 2012 na área 2 e (c) entre 26 e 30 de outubro de 2012 na área 3. A intensidade no dia (Int $(d)$ é dada pela média dos valores de $T_{\max }$ plotados dentro da área cinza claro em cada mapa. Estas áreas em cinza claro mostram a abrangência espacial $(a 1)$ da onda de calor representada pelas estações com registro de $T_{\max } \geq \bar{T}_{\max }+\sigma$. As áreas em cinza escuro mostram abrangência espacial do núcleo mais intenso $\left(a_{2}\right)$ da onda de calor representada pelas estações com registro de $T_{\max } \geq \bar{T}_{\max }+2 \sigma$. A plotagem dos valores de $T_{\max }$ e o traçado das isolinhas delimitando $a_{1}$ e $a_{2}$ foram executados automaticamente pelo software Grid Analysis and Display System (GrADS).

sário assumir esta conclusão sob outras considerações, tais como: (i) a possibilidade, embora baixa, de inversão desta tendência na sequência do período da atual fase fria da ODP, visto que não se sabe a frequência de ocorrência das ondas de calor nos anos anteriores a 1961 da fase fria da ODP de 1947-1976 e (ii) as mudanças nas características de clima local, tais como o efeito da urba- nização nos arredores das estações meteorológicas, embora dificilmente isto tenha ocorrido em número considerável de localizações a ponto de influenciar a metodologia de identificação das ondas de calor proposta neste estudo.

- Em muitos anos com El Niño ou La Niña não ocorrem ondas de calor no Brasil. Porém, para o domínio ao norte 
de $15{ }^{\circ} \mathrm{S}$ há indícios de que, quando identificadas, as ondas de calor ocorrem preferencialmente em anos de El Niño ou neutralidade climática. Já na área 3 , embora a grande maioria das ondas de calor também ocorram em ano de El Niño ou neutro, 6 casos foram identificados em anos de La Niña.

- A duração das ondas de calor é, aproximadamente, a mesma em todo o Brasil. Em média, as ondas de calor duram 4,5 dias na área 1, 4,6 dias na área 2 e 4,7 dias na área 3. Percebeu-se que em geral a duração das ondas de calor passou a ser maior a partir de 2000, lembrando que a partir deste ano o número de ondas de calor também é significativamente maior. Em termos de abrangência espacial isto não é verificado, pois as áreas atingidas pelas ondas de calor são em geral similares ao longo da série estudada. Foi constatado, ainda, que as ondas de calor mais duradoras são, também, as mais abrangentes espacialmente. E, quanto maior a área representada por estações com registro de temperatura máxima superando o valor médio mais um desvio padrão, maior também é a área representada por estações com registro de temperatura máxima superando o valor médio mais dois desvios padrão.

- A intensidade média das ondas de calor é maior na área 1, seguida das áreas 2 e 3 . A onda de calor mais intensa do Brasil foi registrada na área 1. Entre as três ondas de calor mais intensas de cada área, a mais duradoura e abrangente espacialmente ocorreu na área 3. Também se constatou que as ondas de calor mais intensas de cada área ocorreram na fase fria da ODP iniciada em 1999. Analisando o dia mais quente da onda de calor de maior intensidade em cada uma das áreas, verificou-se extremos de temperatura máxima de $39,7{ }^{\circ} \mathrm{C}$ na área 1 , $41,7{ }^{\circ} \mathrm{C}$ na área 2 e $42,6{ }^{\circ} \mathrm{C}$ na área 3 , mostrando que pontualmente o calor mais extremo do Brasil ocorre ao sul de $15^{\circ} \mathrm{S}$ de latitude.

Os resultados deste trabalho deixam claro que o Brasil tem sido atingido por um número consideravelmente maior de ondas de calor a partir do ano 2000. Além disso, há uma real possibilidade de manutenção ou até crescimento deste número, dado os cenários climáticos simulados para o futuro. Por isso, recomenda-se fortemente a contínua realização de estudos sobre este tema e urgente aplicação de medidas mitigadoras, tais como implantação de alertas específicos sobre ondas de calor, emitidos à sociedade pelos centros operacionais de previsão de tempo. Somado a isso, é fundamental o planejamento estratégico dos serviços públicos nos setores médico e sanitário. Os efeitos nocivos do calor intenso à saúde humana somente poderão ser minimizados através de políticas públicas que visem proteger a população, principalmente para as pessoas que desenvolvem atividade laboral, esportiva ou de lazer à céu aberto.

\section{Agradecimentos}

Os autores agradecem ao Instituto Nacional de Meteorologia (INMET) por disponibilizar a série de dados de temperatura máxima diária. Agradecemos aos suportes financeiros do Conselho Nacional de Desenvolvimento Científico e Tecnológico (CNPq) (processos 404201/2013-0 e 449208/2014-1), através dos projetos de pesquisa "O Impacto das Mudanças Climáticas no Estresse Térmico de Trabalhadores a Céu Aberto: Elaboração de uma Proposta de Monitoramento para Cortadores de Cana-de-Açúcar" e "Identificação de Áreas de Risco de Estresse Térmico Provocado por Ondas de Calor no Brasil".

\section{Referências}

ANDRADE, E.M.; SILVA, B.B.; RODRIGUES, M.M.A.; MENDONÇA, M.A.B.; CHAVES, L.C.G. Tendência das temperaturas extremas em região equatorial brasileira: estudo de caso do Ceará. Revista Ciência Agronômica, v. 43, n. 2, p. 262-272, 2012.

BITENCOURT, D.P.; RUAS, A.C.; MAIA, P.A. Análise da contribuição das variáveis meteorológicas no estresse térmico associada a morte de cortadores de cana-de-açúcar. Cadernos de Saúde Pública, v. 28, n. 1, p. 65-74, 2012.

CERNE, S.B.; VERA, C.S. Influence of the intraseasonal variability on heat waves in subtropical South America. Climate Dynamics, v. 36, n. 11, p. 2265-2277, 2011.

CONTI, S.; MELI, P.; MINELLI, G.; SOLIMINI, R.; TOCCACELI, V.; VICHI, M.; BELTRANO, C.; PERINI. L. Epidemiologic study of mortality during the Summer 2003 heat wave in Italy. Environmental Research, v. 98, n. 3, p. 390-399, 2005.

COSTA, D.F.; SILVA, H.R.; PERES, L.F. Identificação de ilhas de calor na área urbana de Ilha Solteira - SP através da utilização de geotecnologias. Engenharia Agrícola, v. 30, n. 5, p. 974-985, 2010.

CUBASCH, U.; MEEHL, G.A.; BOER, G.J.; STOUFFER, R.J.; DIX, M.; NODA, A.; SENIOR, C.A.; RAPER, S.; YAP, K.S. Projections of future climate change. In: HOUGHTON, J. T.; DING, Y.; GRIGGS, D. J.; NOGUER, M.; VAN DER LINDEN, P. J.; DAI, X.; MASKELL, K.; JOHNSON, C. A. Climate change 2001. The scientific basis. Contribution of Working Group I to the Third Assessment Report of the Intergovernmental Panel on Climate Change. Cambridge University Press, Cambridge, UK, pp 881, 2001.

FIRPO, M.A.F.; SANSIGOLO, C.A.; ASSIS, S.V. Climatologia e variabilidade sazonal do número de ondas de calor e de frio no Rio Grande do Sul associadas ao ENOS. Revista Brasileira de Meteorologia, v. 27, n. 1, p. 95-106, 2012.

GASPARRINI, A.; ARMSTRONG, B. The impact of heat waves on mortality. Epidemiology, v. 22, n. 1, p. 68-73, 2011.

HARLAN, S.L.; BRAZEL, A.J.; PRASHAD, L.; STEFANOV, W.L.; LARSEN, L. Neighborhood microclimates and vulnerability to heat stress. Social Science \& Medicine, v. 63, n. 11, p. 2847-2863, 2006.

HESS, J.J.; SAHA, S.; LUBER, G. Summertime acute heat illness in U.S. emergency departments from 2006 through 2010: analysis of a nationally representative sample. Environmental Health Perspectives, v. 122, n. 11, p. 1209-1215, doi: 10.1289/ehp.1306796, 2014. 
HOSHIKO, S.; ENGLISH, P.; SMITH, D.; TRENT, R. A simple method for estimating excess mortality due to heat waves, as applied to the 2006 California heat wave. International Journal of Public Health, v. 55, n. 2, p. 133-137, 2010.

HUANG, W.; KAN, H.; KOVATS, S. The impact of the 2003 heat wave on mortality in Shanghai, China. Science of the Total Environment, v. 408, n. 11, p. 2418-2420, 2010.

INTERGOVERNMENTAL PANEL ON CLIMATE CHANGE (IPCC). Climate Change 2007 - The Physical Science Basis. Contribution of Working Group I to the Fourth Assessment Report of the IPCC. Cambridge Univ. Press, Cambridge, UK, 2007.

INTERGOVERNMENTAL PANEL ON CLIMATE CHANGE (IPCC). Climate change 2001 - The scientific basis. Contribution of Working Group I to the Third Assessment Report of the IPCC. Cambridge Univ. Press, Cambridge, UK, 2001.

JENERETTE, G.D.; HARLAN, S.L.; BRAZEL, A.; JONES, N.; LARSEN, L.; STEFANOV, W. L. Regional relationships between surface temperature, vegetation, and human settlement in a rapidly urbanizing ecosystem. Landscape Ecology, v. 22, n. 3, p. 353-365, 2007.

JONGSIK, H.; KIM, H. Changes in the association between summer temperature and mortality in Seoul, South Korea. International Journal of Biometeorology, v. 57, n. 4, p. 535544, doi: 10.1007/s00484-012-0580-4, 2012.

KAYANO, M.T.; SANSIGOLO, C. Interannual to decadal variations of precipitation and daily maximum and daily minimum temperatures in southern Brazil. Theoretical Applied Climatology, v. 97, n. 1-2, p. 81-90, 2009.

KJELLSTROM, T.; McMICHAEL, A.J. Climate change threats to population health and well-being: the imperative of protective solutions that will last. Global Health Action, v. 6, n. 20816, doi: 10.3402/gha.v6i0.20816, 2013

KJELLSTROM, T.; GABRYSCH, S.; LEMKE, B.; DEAR, K. The 'Hothaps' programme for assessing climate change impacts on occupational health and productivity: an invitation to carry out field studies. Global Health Action, v. 2, doi: 10.3402/gha.v2i0.2082, 2009

LAAIDI, K.; ZEGHNOUN, A.; DOUSSET, B.; BRETIN, P.; VANDENTORREN, S.; GIRAUDET, E.; BEAUDEAU, P. The impact of heat islands on mortality in Paris during the August 2003 heat wave. Environmental Health Perspectives, v. 120, n. 2, p. 254-259, 2012.

LIMA, N.G.B.; GALVANI, E.; FALCÃO, R.M.; CUNHALIGNON, M. Air temperature and canopy cover of impacted and conserved mangrove ecosystems: a study of a subtropical estuary in Brazil. Journal of Coastal Research, v. Special, n. 65, p. 1152-1157, 2013.

LIM, Y.H.; KIM, H.; HONG, Y.C. Variation in mortality of ischemic and hemorrhagic strokes in relation to high temperature. International Journal of Biometeorology, v. 57, n. 1, p. 145-153, doi: 10.1007/ s00484-012-0542-x, 2012.

MANTUA, N.J.; HARE S.R. The Pacific Decadal Oscillation. Journal of Oceanography, v. 58, p. 35-44, 2002.

MANTUA, N.J.; HARE S.R.; ZHANG, Y.; WALLACE, J.M.; FRANCIS, R.C. A Pacific interdecadal climate oscillation with impacts on salmon production. Bulletin of the Ameri- can Meteorological Society, v. 78, n. 6, p. 1069-1079, 1997.

MARENGO, J.A.; CAMARGO, C.C. Surface air temperature trends in Southern Brazil for 1960-2002. International Journal of Climatology, v. 28, n. 7, p. 893-904, 2008.

MARENGO, J.A.; VALVERDE, M.C. Caracterização do clima no Século XX e cenário de mudanças de clima para o Brasil no Século XXI usando os modelos do IPCC-AR4. Revista Multiciência, n. 8, p. 5-28, 2007.

MEEHL, G.A.; TEBALDI, C. More intense, more frequent, and longer lasting heat waves in the $21^{\text {st }}$ century. Science, v. 305, n. 5686, p. 994-997, 2004.

MONTEIRO, A.; CARVALHO, V.; OLIVEIRA, T.; SOUZA, C. Excess mortality and morbidity during the July 2006 heat wave in Porto, Portugal. International Journal of Biometeorology, v. 57, n. 1, p. 155-167, doi:10.1007/ s00484-012-0543-9, 2012.

OSTRO, B.D.; ROTH, L.A.; GREEN, R.S.; BASU, R. Estimating the mortality effect of the July 2006 California heat wave. Environmental Research, v. 109, n. 5, p. 614-619, 2009.

PETKOVA, E.P.; GASPARRINI, A.; KINNEY, P.L. Heat and mortality in New York City since the beginning of the 20th Century. Epidemiology, v. 25, n. 4, p. 554-560, 2014.

RADINOVIÉ, D.; CURIÉ, M. Criteria for heat and cold wave duration indexes. Theoretical and Applied Climatology, v. 107, n. 3-4, p. 505-510, 2012.

RIBEIRO, B.Z.; NUNES, A.B. Variação temporal das ondas de calor na cidade de Pelotas-RS. Anais do IV Encontro Sul Brasileiro de Meteorologia. Pelotas (RS), 2011.

ROBINSON, P.J. On the definition of a heat wave. Journal of Applied Meteorology, v. 40, n. 4, p. 762-775, 2001.

SEMENZA, J.C.; RUBIN, C.H.; FALTER, K.H.; SELANIKIO, J.D.; FLANDERS, W.D.; HOWE, H.L.; WILHELM, J.L. Heat-Related deaths during the July 1995 heat wave in Chicago. The New England Journal of Medicine, v. 335, n. 2, p. 84-90, 1996.

STRECK, N.A.; GABRIEL, L.F.; BURIOL, G.A.; HELDWEIN, A.B.; DE PAULA, G.M. Variabilidade interdecadal na série secular de temperatura do ar em Santa Maria, RS. Pesquisa Agropecuária Brasileira, v. 46, n. 8, p. 781-790, 2011.

TAN, J. Commentary: People's vulnerability to heat wave. International Journal of Epidemiology, v. 37, n. 2, p. 318-320, doi: 10.1093/ije/dyn023, 2008.

TASIAN, G.E.; PULIDO, J.E.; GASPARRINI, A.; SAIGAL, C.S.; HORTON, B.P.; LANDIS, J.R.; MADISON, R.; KEREN, R. Daily mean temperature and clinical kidney stone presentation in five U.S. metropolitan areas: A time-series analysis. Environmental Health Perspectives, v. 122, n. 10, p. 1081-1087, 2014.

TODD, N.; VALLERON, A.J. Space-Time covariation of mortality with temperature: A systematic study of deaths in France, 1968-2009. Environmental Health Perspectives, doi:10.1289/ehp.1307771, 2015.

TRENBERTH, K.E. The definition of E1 Niño. Bulletin of the American Meteorological Society, v. 78, n. 12, p. 27712777, 1997.

All the contents of this journal, except where otherwise noted, is licensed under a Creative Commons Attribution License CC-BY 